\title{
O cuidado de enfermagem a crianças com retardo mental: uma revisão integrativa
}

\author{
Edjancley Teixeira de LIMA ${ }^{1}$ \\ Nayara Medeiros SANTOS ${ }^{2}$ \\ Priscilla Tereza Lopes de SOUZA ${ }^{3}$ \\ Rayssa Naftaly Muniz PINTO ${ }^{4}$ \\ Alynne Mendonça SARAIVA ${ }^{5}$
}

\author{
${ }^{1}$ Acadêmica de Enfermagem pela UFCG, edjancleyteixeira@hotmail.com \\ ${ }^{2}$ Acadêmica de Enfermagem pela UFCG, nayara.jc@hotmail.com \\ ${ }^{3}$ Acadêmica de Enfermagem pela UFCG, priscillasouza_@hotmail.com \\ ${ }^{4}$ Acadêmica de Enfermagem pela UFCG, rayssa.muniz@ $@$ hotmail.com \\ ${ }^{5}$ Orientadora. Professora do Curso de Graduação em Enfermagem da UFCG - Campus Cuité. Paraíba. Mestre em \\ Enfermagem. alynnems@hotmail.com
}

\begin{abstract}
RESUMO: O presente estudo objetiva mostrar a importância do cuidado de enfermagem a crianças com retardo mental. Para isto, foi realizada uma revisão integrativa nas seguintes bases de dados: Scientific Electronic Library Online (Scielo) e Literatura Latino-Americano e do Caribe em Ciências da Saúde (LILACS), sendo utilizados os artigos do período de 2000 a 2011 disponíveis gratuitamente. Localizamos inicialmente 24 artigos dos quais apenas 11 compuseram a mostra desse estudo por se adequarem melhor a temática abordada pelas pesquisadoras. Os resultados apontam que existe uma carência em publicações sobre os cuidados de enfermagem à criança que tem retardo mental. Observamos em nossos estudos que é possível proporcionar uma melhoria na qualidade de vida dessas crianças através da intervenção de enfermagem e de outros profissionais envolvidos no cuidado. É função da equipe multiprofissional minimizar as complicações, oferecer o tratamento adequado a criança e apoio às famílias para uma melhor adaptação às novas condições de vida.
\end{abstract}

PALAVRAS-CHAVE: Retardo mental na infância. Criança. Enfermagem. Cuidados de enfermagem. Relações Profissional-Família.

\section{Integrative review of nursing care for children with mental retardation}

\begin{abstract}
The present study aims to show the importance of nursing care to children with mental retardation. For this, an integrative review was carried in the following databases: Scientific Electronic Library Online (Scielo) and the Literature Latin American and Caribbean Health Sciences (LILACS), using the articles of the period 2000 to 2011 freely available. We located 24 initial articles of which just 11 were included in the study by showing that better are adequate the theme addressed by the researchers. The results show that there is a lack of publications on nursing care to the child who has mental retardation. We observed in our studies that is possible provide a better quality of life of these children through the intervention of nurses and other professionals involved in care. It is function of the multidisciplinary team to minimize complications, provide the appropriate treatment to children and support families to better adapt to new conditions of life.
\end{abstract}

KEYWORDS: Mental retardation in infancy. Children. Nursing. Nursing Care. Professional-Family Relations.

\section{INTRODUÇÃO}

O retardo mental é definido como um funcionamento intelectual abaixo do nível normal, manifestando-se durante o período de desenvolvimento, especialmente em menores de 18 anos de idade. São avaliados alguns fatores como os testes de inteligência mais conhecidos como QI, mas também são utilizados outros métodos para realizar este 
diagnostico como: o surgimento de atraso na motricidade, na fala e na cognição. Pessoas com esse transtorno mental apresentam Quoeficiente de Inteligência (QI) abaixo de 70.

Esse tipo de transtorno neuropsiquiátrico é um dos que mais acomete crianças e adolescentes. Estes indivíduos, muitas vezes apresentam atraso na fala/linguagem, falha na função motora, alteração do comportamento e baixo rendimento escolar ${ }^{(1)}$. As habilidades da vida cotidiana são reduzidas, sua socialização é prejudicada e sua idade mental é pouco desenvolvida $^{(2)}$.

Essa patologia pode ser decorrente de fatores genéticos, de algum dano sofrido pela mãe durante o período gestacional, deficiência nutricional e de oxigenação, pela exposição a produtos tóxicos ou a doenças como rubéola e sarampo no primeiro trimestre da gravidez, crianças com baixo peso ou de mães que fazem uso abusivo de álcool ou algum tipo de droga ${ }^{(3)}$.

O surgimento do retardo mental está associado a vários fatores, inclusive os genéticos que compreendem a síndrome de Down, uma alteração no cromossomo 21; a síndrome do $\mathrm{X}$ frágil decorrente de uma mutação no cromossomo $\mathrm{X}$; a síndrome de Prader-Willi resultante de uma deleção no cromossomo 15; a síndrome do miado de gato, correspondente a deleção de parte do cromossomo 5; além de outras anormalidades menos incidentes como a fenilcetonúria ${ }^{(2)}$.
O diagnóstico precoce dessa doença permite a intervenção médica através de um tratamento com ou sem uso de medicamentos.

O acompanhamento por uma equipe multiprofissional possibilita apoio a essas crianças e suas famílias promovendo a redução de possíveis danos e orientado a qualidade de vida que a mesma pode ter ${ }^{(3)}$.

A classificação do retardo mental varia de acordo com as características apresentadas durante $\mathrm{o}$ crescimento $\mathrm{e}$ desenvolvimento da criança. Podendo apresentar-se de maneira leve, moderada, grave e profunda, sendo o leve o mais incidente entre as pessoas afetadas. O nível de QI também influencia no tipo de retardo, quanto mais grave for o nível dessa comorbidade menor será o QI da criança.

Dependendo do nível do retardo, a criança pode realizar suas atividades diárias através de treinamentos e incorporação de habilidades específicas para a sua condição clínica. Adequações na rotina familiar, informações, participação em grupos de apoio, são estratégias importantes para a melhoria da qualidade de vida desses indivíduos, bem como contribui para a sua inserção na sociedade.

Crianças com retardo mental leve e moderado podem apresentar autoimagem negativa e baixa autoestima, por saberem que são diferentes dos outros, por não ser satisfatórios aos desejos dos pais e familiares e por não estarem inseridos dentro dos padrões de normalidades impostos pela 
sociedade. Desta forma, cabe dentro desse processo, uma abordagem multiprofissional que acolha a criança em todos os seus âmbitos ajudando-a a restabelecer sua estrutura social e sua importância na família.

O Enfermeiro dentro da equipe multiprofissional é responsável pelo acompanhamento tanto na atenção primária quanto nos outros níveis de atenção. Esse processo permite ao profissional uma melhor qualificação nessa área, possibilitando uma assistência individualizada e otimizada.

Para realização dessa assistência qualificada a essas crianças, existem locais específicos que dão suporte e apoio no tratamento dessa patologia como CAPSi (Centro de atenção Psicossocial infantil) e APAE (Associação de Amigos e Pais dos Excepcionais), ONGs sustentadas por empresas privadas e entidades filantrópicas que oferecem uma assistência integral.

Diante do exposto, o trabalho em questão objetiva fazer uma revisão integrativa sobre os cuidados de Enfermagem a crianças com retardo mental.

\section{CONSIDERAÇÕES METODOLÓGICAS}

A revisão integrativa é um tipo de pesquisa que engloba uma procura na base de dados de todos os acervos referentes ou similares à temática ao qual deseja analisar criteriosamente e explicar de uma maneira mais abrangente os fatos que foram encontrados nos estudos ${ }^{(4)}$.

$$
\text { Para a realização deste estudo }
$$
percorremos um caminho metodológico que compreende seis passos, a saber: formulação do tema; coleta do material referente ao tema proposto; avaliação do material; análise do material; elaboração dos resultados e conclusões. Para a continuação do estudo usamos uma questão norteadora: quais são os cuidados de enfermagem e a contribuição dessa equipe na atenção à crianças com retardo mental?

Para a operacionalização dessa pesquisa, a coleta de dados foi realizada pelas pesquisadoras em setembro e outubro de 2011. Procuramos artigos que abordassem a temática em base de dados como Scielo (Scientific Electronic Library Online), Literatura Latino-Americano e do Caribe em Ciências da Saúde (LILACS) uma biblioteca eletrônica que compreende uma coletânea selecionada de periódicos científicos brasileiros, utilizando os Descritores em Ciências da Saúde (DeCS): retardo mental, infância e enfermagem.

Os critérios de inclusão utilizados foram periódicos publicados a partir do ano 2000 até o ano de 2011, que abordassem o transtorno em questão e que relacionasse essa patologia com a Enfermagem. O levantamento do material da pesquisa totalizou 24 artigos, onde destes foram excluídos aqueles que não se enquadravam no estudo em questão. A primeira seleção 
realizada a partir dos títulos/temas dos artigos e de seus resumos, nessa etapa desqualificou 13 artigos, ficando apenas 11 artigos para o aprofundamento da pesquisa. Depois dessa seleção fizemos a leitura por completo dos artigos escolhidos. E posteriormente realizamos a análise crítica desses artigos tomando por base a nossa questão norteadora referente aos cuidados de enfermagem e sua contribuição na atenção à saúde de crianças com retardo mental.

É válido ressaltar que as pesquisadoras levaram em consideração as diretrizes éticas contidas na resolução 311/2007 do Conselho Federal de Enfermagem na qual o CEPE é regulamentado em especial ao que concerne aos capítulos III - Do ensino da pesquisa e da produção técnico - cientifica; e IV - Da publicidade.

\section{RESULTADOS E DISCUSSÃO}

Os dados a seguir referem-se ao levantamento de matérias do estudo em questão, de acordo com os critérios de seleção elaborados pelas pesquisadoras para realização da analise do material referente aos cuidados de enfermagem com as crianças com retardo mental.
TABELA 01 - Distribuição das publicações da área da saúde na atenção à criança com retardo mental em periódicos no período de 2000 a 2011.

\begin{tabular}{llc}
\hline PERIÓDICO & $\mathbf{N}^{\mathbf{0}}$ & $\begin{array}{c}\text { PORCENTAGEM } \\
(\boldsymbol{\%})\end{array}$ \\
\hline Revista Ciência, Cuidado e Saúde & 1 & $9,09 \%$ \\
Acta Scientiarum. Health Science & 1 & $9,09 \%$ \\
Cadernos de Saúde Publica & 1 & $9,09 \%$ \\
Aletheia & 1 & $9,09 \%$ \\
Revista Faipe & 1 & $9,09 \%$ \\
Acta Scientiarum. Health Science & 1 & $9,09 \%$ \\
Cienc Cuid Saude & 1 & $9,09 \%$ \\
Jornal de Pediatria & 2 & $18,18 \%$ \\
TCC/ UEMS & 1 & $9,09 \%$ \\
Revista Inspirar & 1 & $9,09 \%$ \\
\hline Total & $\mathbf{1 1}$ & $\mathbf{1 0 0}$ \\
\hline
\end{tabular}

Após a análise dos 11 artigos podemos perceber uma defasagem na publicação de artigos relacionados à temática de retardo mental na infância, por esse motivo evidenciamos na tabela 01 uma grande diversidade de periódicos que abordam o tema em questão. Apenas dois artigos foram encontrados no mesmo periódico. Para localizarmos um numero de artigos suficiente a elaboração dessa pesquisa perpassamos diversas revistas, tanto da área da enfermagem como de outras categorias da saúde, como a psicologia por exemplo. 
TABELA 02 - Distribuição das publicações da área da saúde na atenção à criança com retardo mental em periódicos de acordo com o tipo de estudo no período de 2000 a 2011.

\begin{tabular}{lcc}
\hline TIPO DE ESTUDO & $\mathbf{N}^{\mathbf{0}}$ & $\begin{array}{c}\text { PORCENTAGEM } \\
(\boldsymbol{\%})\end{array}$ \\
\hline Pesquisa de campo & 4 & $36,3 \%$ \\
Estudo descritivo & 1 & $9,09 \%$ \\
Avaliação da & & \\
equivalência conceitual & 1 & $9,09 \%$ \\
Relato de caso & & $9,09 \%$ \\
Inventário Avaliação & 1 & $9,09 \%$ \\
Ped. Incapacidade & 1 & $\mathbf{1 0 0}$ \\
\hline Revisão Integrativa & 2 & $18,18 \%$ \\
& & \\
\hline Revisão de literature & 1 & $9,09 \%$ \\
\hline
\end{tabular}

A tabela 02 apresenta a caracterização dos artigos que tratam do retardo mental em crianças conforme o tipo de estudo, tornando explicito o tipo de metodologia utilizada. $\mathrm{O}$ maior índice $(36,6 \%)$ trata de uma pesquisa de campo, que obtém dados de profissionais de saúde, crianças e familiares envolvidos nos cuidados de retardo mental. O restante dos dados esta relacionado a informações já existentes em outros arquivos.
TABELA 03 - Distribuição das publicações da área da saúde na atenção à criança com retardo mental em periódicos de acordo com os sujeitos do estudo no período de 2000 a 2011.

\begin{tabular}{lcc}
\hline SUJEITOS DO ESTUDO & $\mathbf{N}^{\mathbf{o}}$ & $\begin{array}{c}\text { PORCENTAGEM } \\
(\boldsymbol{\%})\end{array}$ \\
\hline Profissionais de enfermagem & 1 & $9,09 \%$ \\
Prontuários dos membros APAE & 1 & $9,09 \%$ \\
Pais e mães & 1 & $9,09 \%$ \\
Paciente & 3 & $27,2 \%$ \\
Mulheres & 1 & $9,09 \%$ \\
Outros/revisão & 4 & $36,3 \%$ \\
\hline Total & $\mathbf{1 1}$ & $\mathbf{1 0 0}$ \\
\hline
\end{tabular}

Os dados obtidos na tabela 03 demonstram que $27,2 \%$ dos estudos selecionados foram feitos com os próprios pacientes e os maiores índices (36,3\%) foram baseados em outros estudos já existentes. O restante dos estudos foram distribuídos entre pesquisas com familiares, profissionais, documentos e mulheres que possam vivenciar essas situações.

Observamos que dentre os artigos pesquisados, nenhum relatava o cuidado específico da Enfermagem em crianças nessas condições, e sim a participação de toda a equipe multiprofissional, com enfoque na atenção do psicólogo. Existiam artigos relacionados à assistência de enfermagem sendo que eram pagos, então preferimos usar aqueles que eram disponibilizados nas bases de dados gratuitamente. 


\section{CONSIDERAÇÕES FINAIS}

De acordo com a análise do material, podemos perceber que existe uma defasagem no que se refere à publicação de estudos relacionados ao cuidado de enfermagem aos portadores de retardo mental. E sabemos que tanto este como os demais profissionais da área de saúde são de essencial importância para a assistência a essas crianças.

No decorrer do estudo é evidente que essas crianças são capazes de conseguir ter qualidade de vida mesmo com as limitações que a eles são impostas. Um bom acompanhamento, informações, qualificação das famílias, prática de atividades lúdicas, ajudam bastante no desenvolvimento das habilidades da vida diária desses infantes. Sendo pontos que precisam ser mais abordados em pesquisas e aplicados na prática multidisciplinar, a fim de inserir esses indivíduos na sociedade e diminuir as barreiras que a estes são impostas.

Desta forma, podemos perceber que as crianças acometidas pelo retardo mental necessitam da assistência de uma equipe multidisciplinar, na qual diversos profissionais possam juntar seus conhecimentos e elaborar um plano de cuidados para cada indivíduo. Assim, a Enfermagem como componente primordial da equipe é responsável por promover o atendimento humanizado, através da identificação dos fatores de risco para tentar minimizá-los. O profissional Enfermeiro deve estar atento para os casos de retardo mental, diminuindo as complicações e oferecer o tratamento adequado à criança e o apoio necessário às famílias.

\section{REFERÊNCIAS}

1. Aires M, Roos CM, Gonçalves AVF, Schneider JF, Olschowsky A. Ações em saúde mental às famílias nos diferentes contextos de trabalho: revisão integrativa. Rev Gaúcha Enferm., Porto Alegre (RS) 2010 set;31(3):567-74.

2. Baroneza JE, Faria MJS, Cassola P, Bernardi CC, Silva BP, Costa SC. Afecções neurológicas associadas ao retardo mental em alunos de uma instituição especializada de Londrina, Estado do Paraná. Acta Sci. Health Sci. Maringá (PR), v. 28, n. 1, p. 8791, 2006.

3. Cardozo A, Soares AB. A influência das habilidades sociais no envolvimento de mães e pais com filhos com retardo mental. Aletheia 31, p.39-53, jan./abr. 2010.

4. França DCC, Maria DMC, Correa IPC, Aburad ATT, Aguiar SMHCA. Síndrome do X frágil: Relato de caso. Ver Faipe, Cuiabá (MT) 2011 jan./jul v. 1, n. 1 .

5. Halpern R, Figueiras ACM. Influências ambientais na saúde mental da criança. Jornal de Pediatria Vol. 80, No2(Supl), 2004.

6. Higarashi IH, Pedrazzani JC. O profissional enfermeiro e a criança portadora de deficiência. Revista Ciência, Cuidado e Saúde Maringá (PR), v. 1, n. 1, p. 37-44, 1. sem. 2002. 
7. Losapio MF, Silva LG, Pondé MP, Novaes CM, Santos DN, Argollo N, Oliveira IMS, Brasil HHA. Adaptação transcultural parcial da escala Aberrant Behavior Checklist (ABC), para avaliar aficácia de tratamento em pacientes com retardo mental. Cad. Saúde Pública, Rio de Janeiro, 27(5):909-923, mai, 2011.

8. Moraes, AMSM, Magna LA, Faria APM. Conhecimento de mães sobre fatores de risco e prevenção do retardo mental e/ou defeitos congênitos. Ver Cienc Cuid Saude 2007 Out/Dez; 6(4):401-406.

9. Pacheco R, Matteo JD, Cucolicchio S, Gomes C, Simone MF, Assumpção FB. Inventário de avaliação pediátrica de incapacidade (PEDI):

aplicabilidade no diagnóstico de transtorno invasivo do desenvolvimento e retardo mental. Rev Med Reabil 2010; 29 (1); 9-12.

10. Pereira MFB. Sentimentos dos pais frente ao diagnóstico de síndrome de Down-revisão integrativa de literatura. Universidade Estadual de Mato Grosso do Sul, Dourados (MS) 2010.

11. Sadock BJ, Sadock VA. Compêndio de Psiquiatria. Porto Alegre: Artimed, 2007.

12. Silva NLP, Dessen MA. Síndrome de Down: etiologia, caracterização e impacto na família. Interação em Psicologia. V.6, nº 2, p. 167-76, 2002.

13. Vasconcelos MM. Retardo mental. Jornal de Pediatria - Vol. 80, No2(supl), 2004. 